\title{
Attention-related, cross-modality modulation of somatosensory neurons in primate ventrobasal $(\mathrm{VB})$ thalamus
}

\author{
THOMAS J. MORROW and KENNETH L. CASEY
}

Neurology Research Laboratories, VA Medical Center and Departments of Neurology and Physiology, University of Michigan, Ann Arbor, MI 48105, USA

\begin{abstract}
Attention-related modulation (AM) of the somatosensory responses of single neurons has been demonstrated in the cerebral cortex and medullary dorsal horn, but not in the ventrobasal thalamus. The somatically evoked activity was recorded of single units in the ventral posterior lateral thalamus (VPL) of awake monkeys while they detected the termination of taskrelevant somatic or visual stimuli. Eighteen of 56 somatically responsive VPL neurons are reported that were recorded for enough time for a complete analysis of their responses during both the visual and somatic attention tasks. All neurons were spontaneously active and responded either to innocuous cutaneous (13/18) or deep (5/18) stimuli. Seven neurons (7/18, $38.8 \%$ ) showed AM of somatosensory responsiveness. Two cells $(2 / 7,28.6 \%)$ showed AM only during the visual task, two others $(2 / 7,28.6 \%)$ only during the somatosensory task, and three cells $(3 / 7,42.8 \%)$ showed AM during both tasks. All five cells showing AM during the somatosensory task had enhanced responses to the task-relevant somatic stimulus. In contrast, the somatosensory responses of all five cells showing AM during the visual task were reduced. It is concluded that selective attention is associated with a modality specific modulation of the somatosensory responses of a sub-population of neurons within the primate VPL nucleus.
\end{abstract}

Key words: sensory modulation, attention, somatosensory, ventrobasal thalamus, monkey, single unit recording

\section{Introduction}

An important question in neuroscience concerns the mechanisms by which the central nervous system filters incoming sensory signals and assigns importance or relevance to the specific items of sensory information being received. Our sensory systems continually receive inputs from which information is selected as a basis for action, ignoring all the rest. Cognitive-behavioral studies have shown that attention can influence the perception of visual (Posner et al., 1980; Hillyard and Mangun, 1987; Posner, 1988; Posner and Petersen, 1990) and auditory stimuli (Treisman, 1964; Woldorff and Hillyard, 1991; Woldorff et al., 1993). However, less is known about the influence of attention on somatosensory processing. Cognitive studies involving spatially selective attention (i.e., knowledge of where a stimulus will be applied to the skin) have reported altered detectability of somatic stimuli (Meyer et al., 1963; Post and Chapman, 1991) or no effect on sensory perception (Shiffrin et al., 1973; Posner et al., 1978). Studies examining the influence of cross-modal manipulations of attention (i.e., knowledge of the modality of the stimulus to be presented) have found that direction of attention toward tactile stimuli may (Boulter, 1977) or may not (Shiffrin and Grantham, 1974) modify stimulus detectability. Post and Chapman (1991) have also demonstrated that crossmodality manipulations can modify the detectability of vibrotactile stimuli, an effect the authors attribute to attention. However, other reports suggest that the effectiveness of attentional mechanisms to alter somatosensory perception depends on the specific details of both the stimulus and task (Sathian and Burton, 1991; Whang et al., 1991).

Evoked potential studies have shown that the amplitude of the responses elicited by both painful and non-painful somatosensory stimuli can be modulated by manipulations of attention (Desmedt and Robertson, 1977; Desmedt and Debecker, 1979; Desmedt and Tomberg, 1989; Miltner et al., 1989; Beydoun et al., 1993). At the cellular level, however, evidence of attention-related effects on the somatosensory system is variable across studies. Hyvarinen et al. (1980) found that a small percentage of SI cortical neurons in layers I and II exhibited modulated responses to vibrotactile stimuli during directed attention. More recently, Hsiao et al. (1993) demonstrated attention-related effects on selective form 
processing in both primary and secondary somatosensory cortex. Cross-modal attention has been shown to influence the sensory responses of neurons in the second somatosensory and $7 \mathrm{~b}$ cortical areas of rhesus monkeys (Burton et al., 1997).

It is well accepted that neurons in the ventrobasal complex (VB) of the primate thalamus, which includes the ventral posterior lateral nucleus (VPL), play an important role in the processing of somatosensory information dealing with many stimulusrelated parameters and form a critical relay for input to primary somatosensory cortex (S1). Anatomical studies show that VPL is a principal target for somatosensory projections from the body surface ascending in both the medial lemniscal and spinothalamic pathways (Boivie, 1979; Berkley, 1980; Jones, 1990; Ralston III and Ralston, 1994). Few studies, however, have found attention-related modulation of the firing of VB neurons. Poranen and Hyvarinen (1982) reported that stimulus evoked multiple unit activity in the ventral posterior lateral thalamus showed no significant changes in discharge rate during changes of attention. More recently, Tremblay et al. (1993) also failed to demonstrate attention-related modulation of primate VB neurons in response to cutaneous air puff stimulation of the face. Such findings support the findings of early electrophysiological data which suggest a constant precision in the somatosensory responses of VB neurons (Mountcastle and Henneman, 1952; Poggio and Mountcastle, 1960, 1963; Harris, 1978a, b, 1980). However, other studies report that the evoked activity ofVB thalamic neurons is dynamic and varies with both the level of arousal within the waking state (Casey and Morrow, 1985; Morrow and Casey, 1988, 1992) as well as between sleep and waking (Mariotti et al., 1989).

Because we previously showed that the somatically evoked discharges of some primate VB neurons covary with the level of arousal (Casey and Morrow, 1985; Morrow and Casey, 1988, 1992), we chose to re-examine the somatosensory thalamus with respect to the effects of attention. Accordingly, the aim of this study was to identify somatically activated cells in VPL that exhibited behavioral state-related modulation attributable to cross-modal manipulations of attention. The data presented here demonstrate that the direction of attention toward or away from a somatic stimulus is associated with changes in the sensory responsiveness of neurons in the ventral posterior lateral thalamus of the primate brain.

\section{Methods}

Two young adult male African green monkeys (Cercopithecus aetheops sabaeus) weighing 4.5 and $5.5 \mathrm{~kg}$, respectively, were used for this investigation. Subjects were housed in facilities fully accredited by the American Association for the Accreditation of Laboratory Animal Care (AAALAC). All animals were cared for under strict veterinary supervision in full compliance with the standards in the USPH/NIH Guide for the Care and Use of Laboratory Animals, Revised (1996).

\section{Attention-related behavioral training}

Each monkey was conditioned to sit quietly for up to two and a half hours while partially restrained in a primate chair (Bak Electronics, Inc., Mount Airy, MD, USA) while the experimenter applied repetitive innocuous somatic stimuli to wide areas of the body including the face. The monkeys were rewarded with fruit juice to reinforce good behavior while confined to the chair. Following chair acclimation, each animal was trained to perform a simple operant behavior while attending to either a visual or a somatosensory stimulus. The somatic stimuli used for the purposes of the behavioral paradigm consisted of hair movement by air puffs or mechanical touches of the skin by a von Frey hair. A more detailed description of the somatic stimuli used is presented below. We chose an "absence" paradigm for both the visual and the somatosensory tasks to insure that the animal subject was attending to the task-relevant stimulus (TRS) throughout each trial period. During each trial the subject had to identify when the TRS had terminated (was absent) for correct performance. For directing the animals attention, a behavioral intelligence panel consisting of two colored cue lights (green and yellow), a T-shaped response bar and a red bar-pull indicator light were placed in front of the monkey seated in the primate chair. The green or yellow light was illuminated at the beginning of each trial and indicated which stimulus modality would be relevant for correct performance in the attention paradigm. Figure 1 shows the sequence of events for each of the modality specific attention tasks described below.

For visual attention trials (Fig. 1, right panel), the yellow cue light on signaled to the monkey that a visual trial had been initiated and that attention should be directed toward the yellow light, the taskrelevant stimulus (TRS). For this trial type, the spring-loaded response bar had to be pulled approximately $1.5 \mathrm{~cm}$ toward the monkey and the bar pull maintained during a $5 \mathrm{~s}$ period of yellow cue light illumination. Approximately $162 \mathrm{~g}$ of force was necessary to pull out the bar the $15 \mathrm{~mm}$ necessary to activate a switch, which in turn illuminated the red bar-pull indicator light located in the center of the intelligence panel. This indicator light provided feedback to the monkey that the bar was pulled out sufficiently. Correct performance required that the monkey release the response bar within $1.5 \mathrm{~s}$ of cue light off in order to receive a juice reward. For each visual trial repetition, one per second, innocuous somatic stimuli were delivered to various body regions in the same manner as during chair acclimation procedures. Air puff, von Frey hair and muscle 


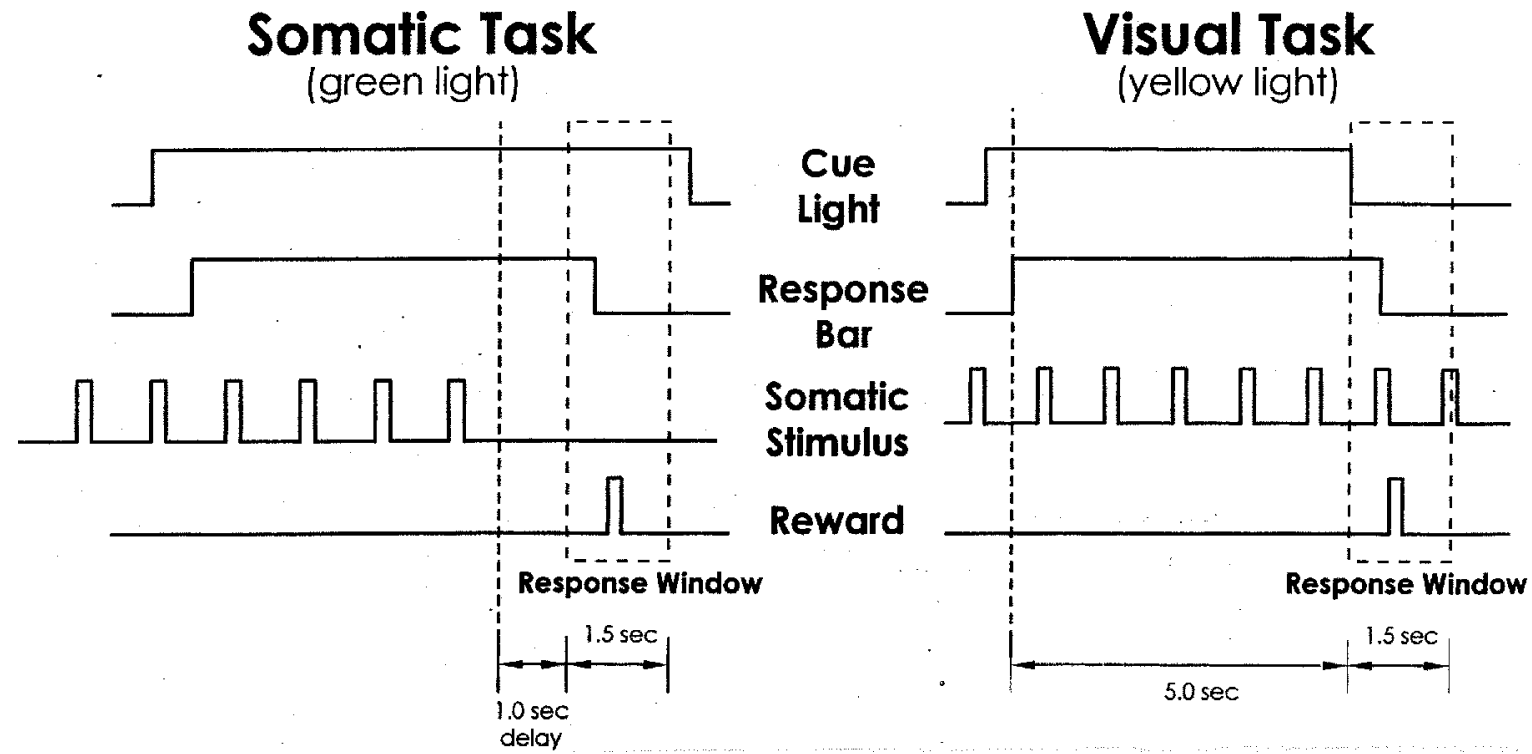

FIGURE 1. Timing of events for individual visual and somatic trials during the attention paradigm. Immediately following termination of the task-relevant cue, the cue light in the visual task or the somatic stimulus in the somatosensory task, the subject has $1.5 \mathrm{~s}$ to release the response lever to receive the reward. Note that for the somatosensory task it would take at least $1 \mathrm{~s}$ (the interval between successive somatic stimuli) to recognize that the somatic stimulation had stopped. Accordingly, we delayed the onset of the correct response window by $1 \mathrm{~s}$. Release of the response lever before cue termination aborts the trial with no reward. Release of the response lever after the correct response window is considered a failed trial with no reward. For both types of task, failed or aborted trials resulted in a 15-30 s time-out period before the next trial could be initiated.

tap stimuli were used during training sessions. For visual trials, somatic stimulation began during the inter-trial period prior to turning on the cue light and continued for several seconds past the reward period. In this way the onset/offset of the somatic stimuli provided no cue for correct visual task performance. The yellow cue light was turned off immediately and no reward given if a trial was aborted due to early bar release. Likewise, no reward was given for failed performance if the bar was held beyond the duration of the response window.

During somatic attention trials (Fig. 1, left), a green cue light signaled the monkey that a somatic trial could be initiated and that attention should be directed toward the somatic TRS, the first somatic stimulus to be presented after bar pull. For these trials, the spring-loaded response bar had to be pulled out toward the monkey and the pull maintained throughout a $5 \mathrm{~s}$ period of somatic stimulation. As described previously, the red indicator light provided feedback to the monkey that the response bar had been pulled out a sufficient distance. During training, calibrated puffs of air, skin touches by von Frey hair or muscle taps served as the TRS. Taskrelevant stimulation was not begun until the bar was pulled, at which time the repetitive somatic stimuli were presented at a rate of one per second. Upon termination of the somatic stimulus, the subject had to release the response bar within a $1.5 \mathrm{~s}$ response window to receive a fruit juice reward. Because it would take at least $1 \mathrm{~s}$ (the interval between successive somatic stimuli) for the monkey to recognize that the TRS had stopped, we delayed the onset of the correct response window by $1 \mathrm{~s}$. Accordingly, bar release was rewarded only if it occurred between 1 and $2.5 \mathrm{~s}$ after presentation of the last somatic TRS. In this way we attempted to keep the visual and somatosensory tasks as similar as possible. In all cases, the green cue light remained on throughout the entire trial and was turned off several seconds following delivery of the reward or by failed performance. Cue light termination had no consequence for the correct performance in this task.

Subjects were trained in the above paradigms until behavioral performance was greater than $85 \%$ correct, after which they were surgically prepared for single unit recording. During unit testing for attention-related modulation, failed performance in the behavioral paradigm accounted for less than $5 \%$ of the total trials presented. The normal inter-trial interval was $15 \mathrm{~s}$ for both visual and somatic trials. However, failed performance resulted in a $30 \mathrm{~s}$ time-out before the next trial was available. Repetitive air puffs, skin touches by von Frey hair or muscle taps were also delivered during the ITP and outside of the above behavioral paradigm when the monkey was alert.

\section{Surgical preparation}

Trained monkeys were anesthetized using halothane $(0.5-1 \%)$, nitrous oxide $(60 \%)$ and oxygen $(40 \%)$, and under aseptic conditions were surgically prepared for single unit recording by making a $15 \mathrm{~mm}$ craniotomy above the stereotaxic coordinates calculated for the exploration of the ventrobasal complex. 
A laboratory designed stainless steel chronic recording chamber was fixed to the skull over this opening using six to eight stainless steel skull screws and acrylic cement. Two skull screws located over frontal cortex served as EEG electrodes and another as ground. All electrode leads were routed to two electrical connectors cemented to the skull posterior to the recording chamber. During post-operative recovery, topical (Mupuracin ${ }^{\mathrm{TM}}$ ointment) and systemic antibiotics (Chloramphenicol, $25 \mathrm{mg} / \mathrm{kg}$, i.m., b.i.d.) were administered routinely to prevent local infection.

\section{Somatic stimuli}

Innocuous somatic stimuli consisted of hair movement, mechanical skin stimulation, joint manipulation and muscle palpation. Hair movement was produced by controlled air puff stimuli delivered using an electromechanical, flow calibrated device held at a fixed distance and angle above the receptive field on the body surface. This device delivered pulsatile constant pressure air puffs with a $0.2 \mathrm{~s}$ duration at a frequency of one puff per second. An airflow sensor mounted near the output orifice was used to detect stimulus onset and duration. Mechanical stimuli of two different intensities ( 14 and $58 \mathrm{~g}$ ) were applied to the skin by calibrated von Frey hair probes. For mechanical skin stimulation during unit recording, stimulus onset is signaled by having the probe complete a low current (less than $1 \mu \mathrm{A}$ d.c.) circuit through the monkey to ground. Noxious stimuli were also tested and included pinprick and skin pinch with serrated forceps sufficient to produce withdrawal. The latter stimuli were painful when applied to the investigator but did not produce tissue damage. Noxious stimuli and joint manipulation were employed only to fully characterize the physiological response properties of each neuron and were not presented during performance in the behavioral tasks. Only innocuous somatic stimuli of constant intensity delivered to the same location within a cell's receptive field ( $R F$ ) were used to assess unit excitability within the attention paradigms.

\section{Single unit recording}

Subjects were allowed to recover for approximately 2 weeks following surgery before the start of unit recording. After surgical recovery, behavioral training was refreshed for 3-5 days before the first recording session to insure that the level of performance was greater than $85 \%$ correct. Between recording sessions, the monkeys were returned to their home cage with the microelectrode in place. A protective outer cap shielded the exposed part of the electrode shaft, which was held firmly in place. Recording sessions were limited to a maximum of $3 \mathrm{~h}$ to minimize the stress produced by restraint and to maintain the cooperation of our animal subjects.
During single unit exploration, Epoxylite ${ }^{\mathrm{TM}}$ insulated stainless steel microelectrodes (8-20 M $\Omega$ impedance at $100 \mathrm{~Hz}$ ) were slowly advanced into the somatosensory thalamus by hydraulic microdrive (Frederick Haer Co.). Unit activity was amplified and filtered using standard techniques and monitored with an oscilloscope and loudspeaker. A timeamplitude window discriminator (Bak and Schmidt, 1977) isolated the activity of neurons from background noise. The signal to noise ratio was typically greater than four to one. The digital pulse output of this device, as well as the digitized outputs from all stimulating devices, task events (cue lights, response bar, reward delivery), movement detector and EEG synchronization detector were fed to the inputs of our laboratory minicomputer system. Discriminator pulses were also fed to a frequency to voltage converter and then to a chart recorder for simultaneous analog display of spike frequency, the EEG, movement detector output, stimulus and task event markers.

Because we previously demonstrated that the general level of arousal can influence unit responsiveness of primate VB neurons (Morrow and Casey, 1992), we initially characterized the basic physiological response properties of each neuron (receptive field, adequate stimulus and level of spontaneous activity) only when the monkey was alert and in a quiet waking state. To this end, the level of arousal was continuously monitored during each experiment and divided into three categories using electroencephalographic and behavioral criteria as previously described (Morrow and Casey, 1992). The quiet waking state was defined as one in which there is no movement, eyes are open, and the EEG exhibits primarily low voltage fast activity. The parameters used to define each behavioral state were monitored using a vibration sensitive accelerometer attached to the primate chair to detect even slight movements of a limb and a microprocessor-based EEG analysis system (Morrow, 1986) which signaled the presence of synchronous high voltage slow wave $(6-17 \mathrm{~Hz})$ activity in the EEG.

Tactile stimuli were routinely applied to the contralateral body surface, as a means of locating units that were not spontaneously active. Only cells exhibiting a response to somatic stimulation were selected for further study. Because we wished to compare unit responses to somatic stimuli of identical intensity applied under different behavioral conditions, during stimulation we restrained the arm and/or leg contralateral to the recording site using restraints built into the primate chair. Under no circumstances did any part of the primate chair or the limb restraints contact the receptive field of a neuron being studied. In addition, test stimuli were not applied to the limb being used by the monkey to perform the behavioral task. This minimized movement artifacts and allowed us to precisely apply the repetitive somatic stimuli. 
After a unit was isolated, a detailed evaluation of receptive field and stimulus-response characteristics was undertaken. The rate and pattern of any spontaneous discharge were also noted. Initial assessment of the adequate stimulus was made by observing unit activity in relation to active and passive movements, gentle palpation of muscles, and response to hair movement and innocuous skin stimulation. The level of responsiveness to somatic stimulation was determined by computing peri-event time histograms and an index of neuronal responsiveness.

\section{State-related analysis}

Unit responsiveness was determined for innocuous somatic stimuli applied to the same point within the receptive field during the visual and somatic attention tasks. Somatosensory responsiveness was also determined for each VB neuron during the inter-trial period. Only the predetermined adequate stimulus for a neuron was used to test for attention-related response differences. During testing for attentionrelated modulation, behavioral trials were presented in blocks of 10-20 trials with both visual and somatic trials presented in a mixed order within each block. These monkeys were highly trained, so they easily switched between the somatic and visual tasks. To minimize investigator induced variability and because it was necessary to apply precise and consistent stimuli to the somatic receptive field, one investigator delivered the somatic stimulus, while a second initiated trial presentation. For attentionrelated testing, peri-event time histograms and an index of unit responsiveness (mean $\delta F \pm$ SEM) were computed for repeated stimuli delivered to the same location in a RF during multiple presentations of either visual or somatic attention trials within the same block.

As described previously (Morrow and Casey, 1992), a quantitative index of neuronal responsiveness (mean $\delta F \pm$ SEM) for multiple stimuli $(N)$ of constant intensity was determined for each neuron. We determined the average discharge frequency $(f)$ in the pre- and post-stimulus periods for each stimulus. Neurons were determined to be responsive to a somatic stimulus if the difference between mean pre- and post-stimulus discharge frequencies $\left(f_{\text {pre }}\right.$ and $f_{\text {post }}$ ) was statistically significant as determined by paired $t$-test ( $p$ " 0.5). The pre-post difference in discharge frequency was then computed for each individual stimulus presentation $\left(\delta F=f_{\text {post }}-f_{\text {pre }}\right)$. Finally, a mean $\delta F \pm$ SEM was computed from the individual values of $\delta F$ of each stimulus during the presentation of multiple $(N)$ stimuli (mean $\delta F=$ $\Sigma(\delta F) / N)$. Computing the discharge frequency on a stimulus-by-stimulus basis allowed us to assess statistically the variability in unit responsiveness during each experimental condition. The lengths of both the pre- and post-stimulus analysis times were determined individually for each cell, and ranged between
80 and $200 \mathrm{~ms}$. For all analyses of a cell's responses, mean $\delta F$ was computed using identical pre- and post-stimulus times and stimulus intensities. The total number of stimulus presentations used to compute the mean $\delta F \pm$ SEM during each behavioral condition for an individual neuron ranged between 35 and 90 stimuli. A mean $\delta F$ of positive sign indicated an increase in unit responsiveness and a negative sign a decreased response to stimulation.

The specific behavioral contexts for which an index of unit responsiveness was computed include the following: (1) the inter-trial period, (2) cue light onset, (3) trials with a correct behavioral response (rewarded trials, visual and somatic), (4) false or aborted trials (unrewarded trials, visual and somatic). Significant differences in unit response between each of the behavioral conditions were determined by comparison of the values of mean $\delta F$ \pm SEM using the $t$-statistic. Average multi-stimulus peri-event time histograms were also computed for stimuli presented during specific behavioral conditions across multiple behavioral trials.

\section{Histological analysis}

Histological verification of recording locations was carried out following several months of recording thalamic unit activity in each subject. At the completion of select electrode penetrations, small electrolytic lesions were made at two locations along the track by passing anodal d.c. current $(50 \mu \mathrm{A}, 20 \mathrm{~s})$ through the electrode tip. At the end of the final recording session, the animals were deeply anesthetized with pentobarbital sodium (100 mg/kg, i.p.) and transcardially perfused with buffered saline, followed by a solution of $10 \%$ formalin in buffered saline. Serial frozen 100 micra coronal sections through the diencephalon and midbrain were cut in the anterior-posterior and vertical planes of the microelectrode tracks, and stained with cresyl violet for later determination of all recording sites. The reconstruction of recording sites was performed by careful comparison of both actual location of the marking lesions on the coronal sections and the calculated stereotaxic coordinates of each electrode placement. Identified recording sites were plotted on standardized drawings of the coronal brain sections.

\section{Results}

Data presented for this study are restricted to 18 of 56 neurons activated by hair movement, skin touch or muscle palpation and histologically verified to be located in the ventrobasal thalamus. These single units were well isolated and were recorded for enough time to completely characterize their physiological responses during behavioral performance in both the visual and somatic attention paradigms (see Methods). As shown in Figure 2, these 18 neurons 


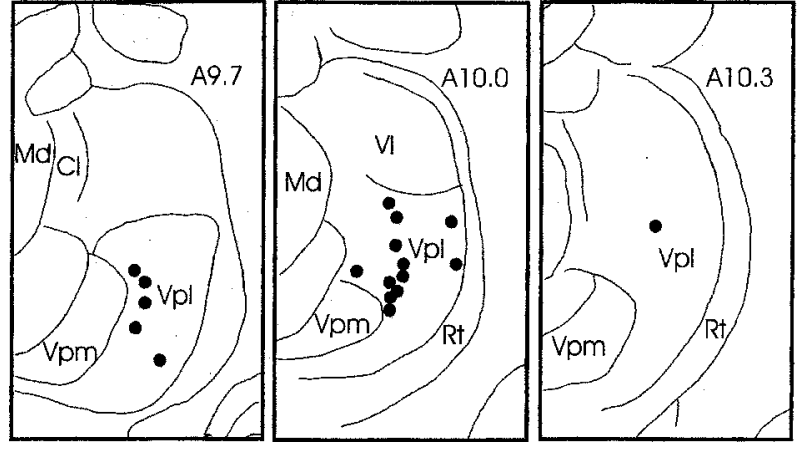

Figure 2. The location of 18 somatically driven units tested for the presence of both AM and ARM. Composite drawings representing sections taken at 9.7, 10.0 and $10.3 \mathrm{~mm}$ anterior to the intra-aural line prepared from photographs of cresyl-violet stained coronal brain sections. Abbreviations: $\mathrm{Vpl}=$ ventral posterior lateral $\mathrm{n} ., \mathrm{Vpm}=$ ventral posterior medial $\mathrm{n} ., \mathrm{Vl}=$ ventral lateral $\mathrm{n} ., \mathrm{Md}=$ medial dorsal $\mathrm{n} ., \mathrm{Cl}=$ central lateral $\mathrm{n}$., $\mathrm{Rt}=$ thalamic reticular $n$.

were located in that region of the ventrobasal thalamus defined as the ventral posterior lateral nucleus (VPL). We do not report on 38 of the neurons that we recorded because technical factors prevented a complete characterization of their responses under all behavioral conditions.

All cells were spontaneously active in the absence of intentional stimulation. Spontaneous discharge rates ranged from $5.4 \pm 6.47$ (SD) spikes per second to $14.4 \pm 17.38$ spikes per second, with a mean rate of $9.11 \pm 3.28$ spikes per second across all cells. The attentional demands of the behavioral task had no significant effect on the level of background discharge for any of the $18 \mathrm{VPL}$ neurons reported here ( $t$-test, $p " 0.05$ ).

Thirteen neurons with cutaneous receptive fields responded exclusively to innocuous mechanical stimulation of the skin or to the movement of hairs.
Receptive fields included discrete areas on the contralateral foot, leg, trunk/back and arm. These units were classified as low threshold (LT), as described previously (Poggio and Mountcastle, 1960, 1963; Harris, 1978a, b, 1980; Morrow and Casey, 1992). The LT neurons recorded here increased their rate of discharge to a single stimulus modality applied to well-defined contralateral cutaneous receptive fields. Five other neurons, which failed to respond to stimulation of cutaneous receptors, increased their discharge to gentle innocuous palpation of muscles or innocuous muscle tap and were classified as DEEP. No cells responded either differentially or exclusively to noxious mechanical stimuli. We did not find units with inhibitory receptive fields. Receptive fields for cutaneous neurons ranged in size from a few guard hairs on the dorsal surface of a toe to several square centimeters for units responsive to the movement of hairs on the contralateral surface of the back and trunk. Because measuring the area of a receptive field in awake behaving animals is difficult, we did not attempt to measure changes in RF size during performance in the attention tasks.

\section{Attention-related modulation (AM)}

After initial identification of the adequate stimulus modality and receptive field, each VB neuron was quantitatively assessed for the presence or absence of response modulation during changes in behavioral state. Seven somatically driven VB neurons (7/18, $38.8 \%$ ) showed attention-related modulation (AM) while the monkey performed either the visual or somatosensory task (see Table 1). Two AM neurons showed a modulation of their cutaneous sensory responses only during the visual task, two others exhibited AM only during the somatosensory task, and three cells exhibited AM during both tasks. All seven AM neurons consistently showed a direction of

TABLE 1. Properties of VB units showing modulation of somatosensory responsiveness during attention

\begin{tabular}{|c|c|c|c|c|c|c|c|}
\hline \multirow[b]{2}{*}{ Unit } & \multirow{2}{*}{$\begin{array}{c}\mathrm{AM} \\
\text { somatic } \\
\text { task }\end{array}$} & \multirow{2}{*}{$\begin{array}{c}\mathrm{AM} \\
\text { visual } \\
\text { task }\end{array}$} & \multicolumn{3}{|c|}{ Mean $\delta F($ spikes $/ \mathrm{s} \pm \mathrm{SD})$} & \multirow[b]{2}{*}{ Receptive field } & \multirow[b]{2}{*}{$\begin{array}{l}\text { Adequate } \\
\text { stimulus }\end{array}$} \\
\hline & & & $\begin{array}{l}\text { Inter-trial } \\
\text { period }\end{array}$ & $\begin{array}{c}\text { Somatic } \\
\text { task }\end{array}$ & $\begin{array}{l}\text { Visual } \\
\text { task }\end{array}$ & & \\
\hline 1 & $\uparrow$ & - & $75.02 \pm 16.75$ & $110.71 \pm 26.37$ & $79.77 \pm 31.81$ & great toe & hair movement \\
\hline 2 & $\uparrow$ & - & $65.67 \pm 32.44$ & $102.95 \pm 40.18$ & $77.25 \pm 30.65$ & dorsum of foot & skin touch \\
\hline 3 & - & $\downarrow$ & $27.09 \pm 36.67$ & $25.50 \pm 29.87$ & $14.40 \pm 33.81$ & calf & muscle press \\
\hline 4 & - & $\downarrow$ & $42.05 \pm 14.58$ & $45.06 \pm 15.41$ & $29.35 \pm 13.44$ & trunk-back & hair movement \\
\hline 5 & $\uparrow$ & $\downarrow$ & $44.67 \pm 18.93$ & $55.81 \pm 21.33$ & $35.26 \pm 19.81$ & trunk-chest & hair movement \\
\hline 6 & $\uparrow$ & $\downarrow$ & $63.47 \pm 11.67$ & $72.33 \pm 14.40$ & $58.45 \pm 14.16$ & knee & hair movement \\
\hline 7 & $\uparrow$ & $\downarrow$ & $38.45 \pm 16.2$ & $47.57 \pm 13.56$ & $29.51 \pm 13.61$ & hip & hair movement \\
\hline
\end{tabular}

\footnotetext{
$\uparrow=$ increased unit responsiveness.

$\downarrow=$ decreased unit responsiveness.

$-=$ no change in responsiveness.
}

BOLD font $=$ significant difference from inter-trial period $(p<0.05$, two-tailed $t$-test $)$. 

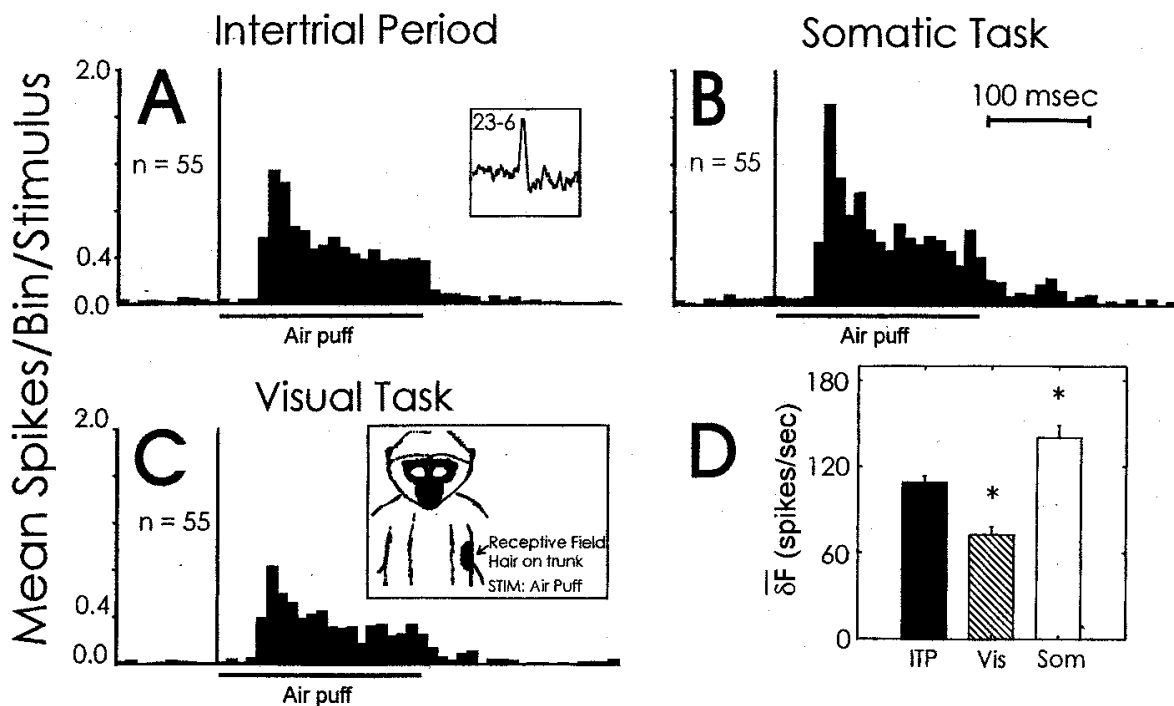

FIGURE 3. Averaged multitrial peri-event time histograms showing the responses of a VPL thalamic neuron to hair movement (air puff) within a receptive field on the contralateral trunk during performance in the somatosensory attention task. (A) Responses obtained during the inter-trial period. (B) Responses obtained during correct performance in the somatic attention task. (C) Responses obtained during the visual attention task. As compared with the inter-trial period (A), unit discharges elicited by air puff are increased (B) when the task-relevant cue is the somatic stimulus used to test unit excitability. When the task-relevant stimulus is the yellow cue light (visual task), responses to somatic stimuli are reduced (C). The number of stimuli $(n)$ used for histogram computation is shown in each of the respective panels. The vertical line from top to bottom of horizontal axes in each histogram represents the onset of each somatic stimulus (A-C). The bar shown below the horizontal axis indicates the duration of the somatic stimulus. The bar graph (D) compares the index of responsiveness (mean $\delta F$, spikes/s with SEM) computed for stimuli delivered during the inter-trial period (It) and during the visual (Vis) and somatic (Som) attention tasks. Statistical differences in response were determined by separately comparing the mean $\delta F$ of each test condition with that of each other using the $t$-statistic. Differences were considered statistically significant for values of $p$ " 0.02 based on the Bonferroni correction for multiple comparisons among conditions and are indicated by the asterisk ${ }^{\star}$ ). INSETS: (A) an oscilloscope tracing of a single discharge from this unit; (C) the location of the cutaneous receptive field on the contralateral trunk.

change in mean $\delta F$, which was tightly coupled to the specific behavioral task (visual or somatic) during which the data was acquired. There was no significant modulation of responses to test stimuli delivered during failed trials (incorrect responses) as compared with the control or inter-trial periods for either the somatic or visual tasks. None of the neurons we recorded responded to specific task parameters alone (cue light or bar pull) in the absence of a somatic test stimulus. Differences in pre- vs post-event discharge rates averaged $1.36 \pm$ $1.33 \mathrm{spikes} / \mathrm{s}$ when the bar pull was used as the histogram trigger event and $0.37 \pm 1.11$ spikes/s for the cue light.

Somatosensory task When compared with responses for stimuli presented during the inter-trial period, all five of the seven AM neurons (71.4\%) showed a 20.9 $\pm 3.5 \%$ SEM increase in somatosensory responsiveness during rewarded somatic trials, when attention to the somatic test stimulus was required for correct performance in the task. Three of the five cells showing somatic AM also exhibited an average 17.4 $\pm 4.7 \%$ SEM reduction in the somatic evoked responses elicited during performance in the visual task as compared with the inter-trial period. The remaining two units did not exhibit response mod- ulation during visual attention (Table 1). Figure 3 illustrates the enhanced somatosensory responsiveness of one VB neuron during attention to the taskrelevant somatic stimulus and depression of responsiveness during attention to the task-relevant cue light in the visual task for correctly performed (rewarded) trials. The modulated responses during somatic and visual attention are evident in both perievent time histograms, as well as in the accompanying bar graph comparing mean $\delta F$ for the inter-trial period (ITP) with that during the somatic (Som) and visual (Vis) test periods.

Visual task The somatosensory responses of five of the seven $(71.4 \%)$ thalamic AM neurons were all reduced on an average of $25.85 \pm 6.3 \%$ SEM during the visual task as compared with the responses obtained during the inter-trial period (Table 1). Three of the five AM neurons showing response depression during the visual task also increased their responsiveness to stimuli presented during the somatic task. Two other neurons (2/5) showing AM during visual trials did not exhibit response enhancement during the somatic trial. Figure 4 illustrates depression of somatosensory responses for one thalamic AM neuron during visual task performance. In contrast to the neuron in Figure 3, which was 


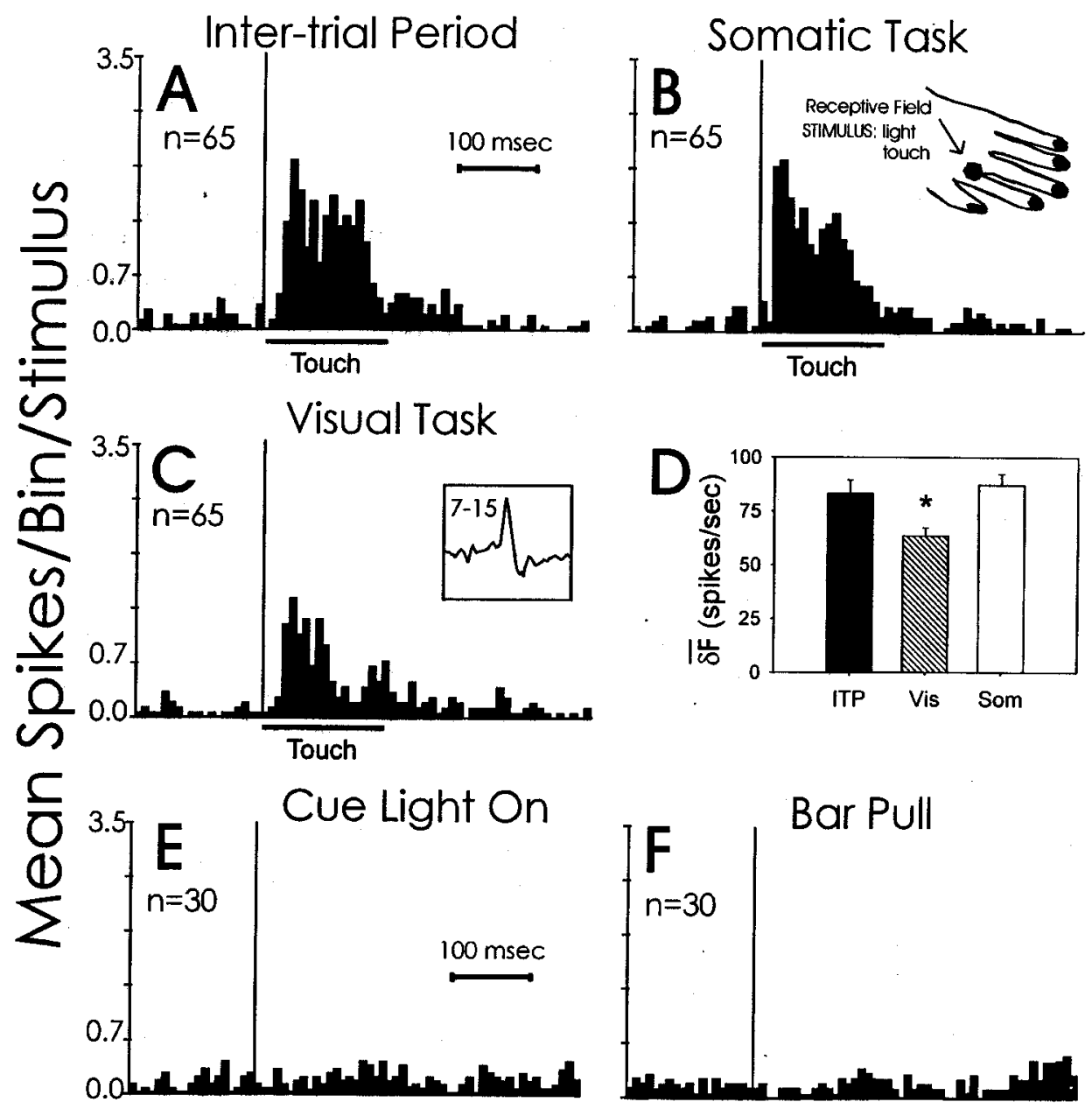

FIGURE 4. Averaged multitrial peri-event time histograms showing the responses of a VPL thalamic neuron to repetitive touch stimulation applied to a receptive field on the contralateral foot during performance in the somatosensory and visual attention tasks. As compared with the inter-trial period (A), unit discharges elicited by touch stimulation are unchanged (B) when the TRS is the somatic touch stimulus. (C) Reduction in unit responsiveness to touch stimuli delivered during visual task performance. (D) Bar graph comparing the index of responsiveness (mean $\delta F$, spikes/s with SEM) computed for stimuli delivered during the inter-trial period (It) with that computed during the visual (Vis) and somatic (Som) attention tasks. Statistical differences in response were determined by comparing the values of mean $\delta F$ for each test period using the $t$-statistic corrected for multiple comparisons (Bonferroni correction, $p " 0.02$ ). Significant differences in the ITP are indicated by the asterisk $\left(^{\star}\right)$. Note that when cue light onset $(E)$ or bar pull $(F)$ were used to trigger histogram generation instead of a somatic stimulus, these task events did not in themselves evoke a change in unit discharge. The vertical line from top to bottom of horizontal axes in each histogram represents the onset of each somatic stimulus (A-C) or task event (E and F). The bar shown below the horizontal axis indicates the duration of the somatic stimulus. The number of trigger events $(n)$ used for histogram generation is shown in each of the respective panels. INSETS: (B) the location of the muscle receptive field on the contralateral leg; (C) oscilloscope tracing of a single discharge from this unit.

modulated during both the somatic and visual attention, the responsiveness of this unit showed AM only during correct performance of visual trials. Figure 4 also shows the lack of response of this neuron to either cue light onset or bar pulls alone when no somatic stimulus was applied to the receptive field.

We did not observe an association between the presence of $A M$ and the stimulus modality required for activation of any cell, but our sample size is small. Table 1 shows the stimulus modalities of the seven VB showing AM neurons recorded in this study. These data suggest that attention-related modulation is likely to occur in VB neurons receiving input from any stimulus modality. Similarly, it appeared that AM occurred without regard to the location of the receptive field.

\section{Discussion \\ Comparison with other studies}

Our data demonstrates that selective attention is associated with modality specific modulation of the responses of single somatosensory neurons within the ventrobasal complex of the primate thalamus. Thus, the somatosensory responses of some VPL neurons were enhanced when the somatic stimulus 
was relevant for successful task performance. Conversely, when the animal was asked to attend to visual stimuli (the cue light in the visual task), the somatically evoked discharges of some VPL cells were attenuated.

Other laboratories found no influence of attention on the firing of neurons in either the ventrobasal thalamus or in its major projection area, the primary somatosensory cortex (SI). Hyvarinen et al. (1980) trained monkeys to press a bar upon cessation of a vibratory stimulus delivered within the receptive field of single cortical neurons in S1. A light cue was used to distinguish rewarded ("relevant") from nonrewarded ("irrelevant") trials. Although they found a few cells in cortical laminae I and II that showed increases in response during relevant, as compared with irrelevant trials, most neurons responded similarly during both types of trials. Subsequent experiments from this laboratory also failed to show significant effects of attention on the multiple unit responses of neurons in either somatosensory cortex or VPL (Poranen and Hyvarinen, 1982).

Differences in behavioral paradigms may offer one explanation for these contrasting results. In the study of Poranen and Hyvarinen (1982), the monkey was cued about whether a trial was relevant or not relevant, but the animal may have attended to the somatic stimulus in each case. This experimental design would test only the effect of the opportunity for reward on unit activity. In our study, we tested for differences in unit responsiveness using cross-modal manipulations of attention. The animals' attention was directed toward either the somatic or the visual stimulus, and all trials were relevant in that the monkey could be rewarded for correct performance. During visual trials, the cue light served as a cross-modality distracter. We looked for differences between the unit responses obtained during visual and somatic trials and compared activity obtained during the respective behavioral tasks with that obtained during the inter-trial period.

Other differences between the behavioral protocols might also explain our findings. Our animals had to initiate and maintain an operant behavior (bar pull) throughout the entire trial period, and release the bar only when the task-relevant somatic or visual stimulus was terminated. This requirement insured that our subjects constantly attended to the task-relevant stimulus (visual or somatic) during the entire time of unit testing. This design differs from the experimental paradigm of Poranen and Hyvarinen (1982) which required an operant behavior (bar press) only at the cessation of the stimulus and provided less behavioral control during testing. For example, their monkeys may not have attended to either the relevant or irrelevant somatic test stimulus throughout the period of stimulation, because correct performance required only that they press the bar when vibration terminated at the end of the task.
Tremblay et al. (1993b) also reported that attentional changes between tactile and visual modalities fail to alter air puff evoked responses in ventral posterior medial (VPM) thalamic neurons of awake monkey. They concluded that attention must alter sensory perception of tactile information either at cortical levels or in subcortical extralemniscal pathways that do not include the ventrobasal thalamus. In their study, monkeys detected an increase in stimulus intensity (air puff or visual) above a constant baseline intensity of the same type of stimulus; they did not test the effect of cross-modality distraction on thalamic unit responses. In our experiments, monkeys had to detect the termination of either a continuous visual stimulus (a cross-modal distracter) or a series of repetitive somatic stimuli. Human psychophysical studies (Sathian and Burton, 1991; Whang et al., 1991) have shown that attentional effects are highly dependent on stimulus and task. It is possible that differences in the attentional demands of our behavioral paradigm as compared with that of Tremblay et al. (1993) may be responsible for the disparity between the finding of these studies.

There is behavioral, imaging and electrophysiological evidence that support the presence of AM within cortical and subcortical sensory structures. Human psychophysical data show that both tactile (Boulter, 1977; Post and Chapman, 1991; Sathian and Burton, 1991; Whang et al., 1991) and pain perception (McCaul and Haugtvedt, 1982; Bushnell et al., 1985; Miron et al., 1989) in humans are markedly altered by attention. Studies mapping human somatosensory evoked potentials (SEPs) during selective attention have also shown changes in SEP amplitude or latency at cortical (Desmedt and Tomberg, 1989; Garcia-Larrea et al., 1991; Beydoun et al., 1993) and thalamic (Velasco and Velasco, 1975; Velasco et al., 1980) levels. Similar findings have also been reported in animal experiments (Ciancia et al., 1988). Early work by HernandezPeon and co-workers suggested that the filtering of afferent input during attention occurs at subcortical levels within a sensory pathway (Hernandez-Peon et al., 1956a, b; Hernandez-Peon, 1959). Studies using positron emission tomographic techniques (PET) have described changes in the pattern of regional cerebral blood flow within somatosensory cortex during attention to tactile stimuli (Roland, 1982; Meyer et al., 1991). Other brain imaging studies have also shown attention-related changes in the activation of subcortical structures including the superior colliculus (Rees et al., 1997), midbrain reticular formation and thalamic intralaminar nuclei (Kinomura et al., 1996). Recently, Burton et al. (1997) described extensive cue-dependent cross-modal attention effects on neuronal firing patterns in the second somatosensory cortical area of rhesus monkeys. Hsiao et al. (1993) also demonstrated modulation of unit responses in primate SI and SII somatosensory cortices during a task that required 
sustained attention to tactile or visual stimuli. In this study, monkeys were trained to detect a raised letter scanning across the finger (tactile discrimination task) or a dimmed square on a video monitor (visual detection task). They found significant differences in the discharge rates evoked by raised letters during the two tasks in approximately $50 \%$ of neurons in SI cortex and about $80 \%$ of neurons in SII cortex. These findings are somewhat analogous to our own findings in the thalamus, because the detection of attentional effects depended upon both sustained attention and the behavioral relevance of the somatic stimulus.

\section{Mechanisms of $A M$}

Several neural mechanisms could be responsible for $\mathrm{AM}$ of thalamic somatosensory responses. The modulation of thalamic sensory responses during attention could be due to changes in activity within the subthalamic structures in the ascending somatosensory pathway projecting to VB as well as mechanisms intrinsic to the thalamus. Evidence from our own as well as other laboratories suggests that behaviorrelated modulation may include mechanisms operating at or below the thalamus (Hoffman et al., 1981; Murray and Cowan, 1990; Shin and Chapin, 1990a, b; Morrow and Casey, 1992; Shin et al., 1993). Intra-thalamic connections with the adjacent thalamic reticular nucleus (RT) exert powerful controls over the discharge of neurons in VB and the lateral geniculate nucleus (Yingling and Skinner, 1976; Sugitani, 1979; Mushiake et al., 1984; French et al., 1985; Harris, 1987; DeBiasi et al., 1988; Fourment et al., 1988; Chen et al., 1992; Warren and Jones, 1994). It has been suggested that these reciprocal connections between RT and the thalamic sensory nuclei play a major role in selective attention (Crick, 1984; Steriade et al., 1986; Guillery et al., 1998). Behavioral, anatomical and electrophysiological studies crossing all sensory modalities, suggest that corticothalamic connections, including those from $\mathrm{S} 1$, anterior cingulate, and posterior parietal cortices may be key participants in a complex neural network for directed attention (Mesulam, 1981; Hurlbert and Poggio, 1985; Posner and Petersen, 1990; Kinchla, 1992; Posner and Driver, 1992). Previous investigations have demonstrated that functional blockade (Yuan et al., 1986) or stimulation (Shin and Chapin, 1990a) within S1 cortex can also profoundly alter the sensory evoked discharges of VB neurons (Yuan et al., 1985; Ghosh et al., 1994). It is therefore likely that the modulation of thalamic responses reported here might be the result of a combination of cortical and subcortical influences on VPL neurons.

\section{Acknowledgements}

This work was supported by grants to Dr Morrow from the Department of Veterans Affairs, the
National Institutes of Health (RO1-NS21104), and Air Force Office of Scientific Research (AFOSR850286). We thank Patricia Walsworth for her invaluable assistance in the training of our monkeys and with single unit recording. Our thanks also go to $\mathrm{Mr}$ Revius Williams, Director of Ann Arbor VAMC Animal Research Facility for his assistance with animal preparation. We also express our gratitude to Dr Pamela E. Paulson for her insightful comments during the preparation of this manuscript.

\section{References}

BAK, M.J., and E.M. Schmidt (1977) An improved timeamplitude window discriminator. IEEE Trans Biomed Eng 5: 486-489.

Berkley, K.J. (1980) Spatial relationships between the terminations of somatic sensory and motor pathways in the rostral brainstem of cats and monkeys. I. Ascending somatic sensory inputs to lateral diencephalon. $f \operatorname{Comp}$ Neurol 193: 283-317.

Beydoun, A., T.J. Morrow, J.F. Shen, and K.L. Casey (1993) Variability of laser-evoked potentials: Attention, arousal and lateralized differences. Electroencephalogr Clin Neurophysiol Evoked Potentials 88: 173-181.

Borvie, J. (1979) An anatomical reinvestigation of the termination of the spinothalamic tract in the monkey. $\mathcal{F}$ Comp Neurol 186: 343-370.

Boulter, L.R. (1977) Attention and reaction times to signals of uncertain modality. $\mathcal{f}$ Exp Psychol 3: 379-388.

Burton, H., R.J. Sinclair, S.Y. Hong, J.R. Pruett, Jr., and K.C.WHANG (1997) Tactile-spatial and cross-modal attention effects in the second somatosensory and $7 \mathrm{~b}$ cortical areas of rhesus monkeys. Somatosens Mot Res 14: 237-267.

Bushnell, M.C., G.H. Duncan, R. Dubner, R.L. Jones, and W. Maixner (1985) Attentional influences on noxious and innocuous cutaneous heat detection in humans and monkeys. F Neurosci 5: 1103-1110.

Casey, K.L., and T.J. Morrow (1985) Arousal-related changes in the response of ventral posterior thalamic neurons to somatic and spinothalamic stimulation in the awake monkey. In Advances in Pain Research and Therapy, H.L. Fields, R. Dubner, and F. Cervero, eds., pp. 285-294, Raven Press, New York.

Chen, S., V. Raos, and M. Bentivoglio (1992) Connections of the thalamic reticular nucleus with the contralateral thalamus in the rat. Neurosci Lett 147: 85-88.

Ciancia, F., M. Maitte, J. Honore, B. Lecoutre, and J.-M. Coquery (1988) Orientation of attention and sensory gating: an evoked potential and reaction time studying in cat. Exp Neurol 100: 274-287.

Crick, F. (1984) Function of the thalamic reticular complex: The searchlight hypothesis. Proc Natl Acad Sci USA 81: 4586-4590.

Debiasi, S., C. Frassoni, and R. Spreafico (1988) The intrinsic organization of the ventroposterolateral nucleus and related reticular thalamic nucleus of the rat: A double-labeling ultrastructural investigation with $\gamma$-aminobutyric acid immunogold staining and lectinconjugated peroxidase. Somatosens Res 5: 187-204.

Desmedt, J.E., and J. Debecker (1979) Wave form and neural mechanism of the decision P350 elicited without pre-stimulus CNV or readiness potential in random sequences of near-threshold auditory clicks and finger stimuli. Electroencephalogr Clin Neurophysiol 47: 648-670. 
Desmedt, J.E., and D. Robertson (1977) Differential enhancement of early and late components of the cerebral somatosensory evoked potentials during forced-paced cognitive tasks in man. $\mathcal{F}$ Physiol (Lond) 271: 761-782.

Desmedt, J.E., and C. Tomberg (1989) Mapping early somatosensory evoked potentials in selective attention: critical evaluation of control conditions used for titrating by difference the cognitive P30, P40, P100 and N140. Electroencephalogr Clin Neurophysiol 74: 321-346.

Fourment, A., J.C. Hirsch, and M.E. Marc (1988) Reticular control of thalamic transmission during behavioral states: a study in dorsal lateral geniculate nucleus relay neurons of the cat. Exp Neurol 100: 305-321.

French, C.R., A.J. Sefton, and A. Mackay-Sim (1985) The inhibitory role of the visually responsive region of the thalamic reticular nucleus in the rat. Exp Brain Res 57: 471-479.

Garcia-Larrea, L., H. Bastuji, and F. Mauguiere (1991) Mapping study of somatosensory evoked potentials during selective spatial attention. Electroencephalogr Clin Neurophysiol 80: 201-214.

Ghosh, S., G.M. Murray, A.B. Turman, and M.J. Rowe (1994) Corticothalamic influences on transmission of tactile information in the ventroposterolateral thalamus of the cat: Effect of reversible inactivation of somatosensory cortical areas I and II. Exp Brain Res 100: $276-286$

Guillery, R.W., S.L. Feig, and D. Lozsadi (1998) Paying attention to the thalamic reticular nucleus. Trends $\mathrm{Neu}$ rosci 21 : 28-32.

HARris, F.A. (1978a) Functional subsets of neurons in somatosensory thalamus of the cat. Exp Neurol 58: $149-170$.

HARRIS, F.A. (1978b) Regional variations of somatosensory input convergence in nucleus VPL of cat thalamus. Exp Neurol 58: 171-189.

Harris, F.A. (1980) Wide-field neurons in somatosensory thalamus of domestic cats under barbiturate anesthesia. Exp Neurol 68: 27-49.

Harris, R.M. (1987) Axon collaterals in the thalamic reticular nucleus from thalamocortical neurons of the rat ventrobasal thalamus. F Comp Neurol 258: 397-406.

Hernandez-Peon, R. (1959) Centrifugal control of sensory inflow to the brain and sensory perception. Acta Neurol Latinoamer 5: 279-298.

Hernandez-Peon, R., H. Scherrer, and M. Jouvet (1956a) Modification of electric activity in the cochlear nucleus during attention in unanesthetized cats. Science 123: $331-332$.

Hernandez-Peon, R., H. Scherrer, and M. Velasco (1956b) Central influences on afferent conduction in the somatic and visual pathways. Acta Neurol Latinoamer 2: $8-22$.

Hillyard, S.A., and G.R. MANGun (1987) Sensory gating as a physiological mechanism for visual selective attention. Electroencephalogr Clin Neurophysiol Suppl 40: 61-67.

Hoffman, D.S., R. Dubner, R.L. Hayes, and T.P. Medlin (1981) Neuronal activity in medullary dorsal horn of awake monkeys trained in a thermal discrimination task. I. Responses to innocuous and noxious thermal stimuli. f Neurophysiol 46: 409-427.

Hsiao, S.S., D.M. O-Shaughnessy, and K.O. Johnson (1993) Effects of selective attention on spatial form processing in monkey primary and secondary somatosensory cortex. F Neurophysiol 70: 444-447.

Hurlbert, A., and T. Poggio (1985) Spotlight on attention. TINS 8: 309-311.

Hyvarinen, J., A. Poranen, and Y. Jokinen (1980) Influence of attentive behavior on neuronal responses to vibration in primary somatosensory cortex of the monkey. $f$ Neurophysiol 43: 870-882.
Jones, E.G. (1990) The Thalamus, Plenum, New York.

Kinchla, R.A. (1992) Attention. Ann Rev Psychol 43: 711-742.

Kinomura, S., J. Larsson, B. Gulyás, and P.E. Roland (1996) Activation by attention of the human reticular formation and thalamic intralaminar nuclei. Science 271: $512-515$.

McCaul, K.D., and C. Haugtvedt (1982) Attention, distraction, and cold-pressor pain. I Pers Soc Psychol 43: 154-162.

Mariotti, M., A. Formenti, and M. Mancia (1989) Responses of VPL thalamic neurones to peripheral stimulation in wakefulness and sleep. Neurosci Lett 102: $70-75$.

Mesulam, M.M. (1981) A cortical network for directed attention and unilateral neglect. Ann Neurol 10: 309-325.

Meyer, E., S.S.G. Ferguson, R.J. Zatorre, B. Alivisatos, S. Marrett, A.C. Evans, and A.M. Hakim (1991) Attention modulates somatosensory cerebral blood flow response to vibrotactile stimulation as measured by positron emission tomography. Ann Neurol 29: 440-443.

Meyer, V., C.G. Gross, and H.-L. Teuber (1963) Effect of knowledge of the site of stimulation on the threshold for pressure sensitivity. Percept Mot Skills 16: 637-640.

Miltner, W., R. Johnson, C. Braun, and W. Larbig (1989) Somatosensory event-related potentials to painful and non-painful stimuli: effects of attention. Pain 38: 303-312.

Miron, D., G.H. Duncan, and M.C. Bushnell (1989) Effects of attention on the intensity and unpleasantness of thermal pain. Pain 39: 345-352.

Morrow, T.J. (1986) A microprocessor based device for real-time detection of synchronized EEG activity. Brain Res Bull 16: 439-442.

Morrow, T.J., and K.L. Casey (1988) Modulation of the spontaneous and evoked discharges of ventral posterior thalamic neurons during shifts in arousal. Brain Res Bull 21: 433-438.

Morrow, T.J., and K.L. Casey (1992) State related modulation of thalamic somatosensory responses in the awake monkey. I Neurophysiol 67: 305-317.

Mountcastle, V.B., and E. Henneman (1952) The representation of tactile sensibility in the thalamus of the monkey. f Comp Neurol 97: 409-440.

Murray, C.W., and A. Cowan (1990) Formalin nociception in the mouse does not lead to increased spinal serotonin turnover. Neurosci Lett 108: 132-137.

Mushiake, S., A. Shosaku, and Y. Kayama (1984) Inhibition of thalamic ventrobasal complex neurons by glutamate infusion into the thalamic reticular nucleus in rats. F Neurosci Res 12: 93-100.

NIH Guide for the Care and Use of Laboratory Animals (1996) National Academy Press, Washington, DC.

Poggio, G.F., and V.B. Mountcastle (1960) A study of the functional contributions of the lemniscal and spinothalamic systems to somatic sensibility. Bull fohns Hopkins Hosp 108: 266-316.

Poggio, G.F., and V.B. Mountcastle (1963) The functional properties of ventrobasal thalamic neurons studied by unanesthetized monkeys. I Neurophysiol 26: 775-806.

Poranen, A., and J. Hyvarinen (1982) Effects of attention on multiunit responses to vibration in the somatosensory regions of the monkey's brain. Electroencephalogr Clin Neurophysiol 53: 525-537.

Posner, M.I. (1988) Structures and functions of selective attention. In Clinical Neuropsychology and Brain Function: Research, Measurement, and Practice, T. Boll and B. Bryant, eds., pp. 169-201, American Psychological Association. 
Posner, M.I., and J. Driver (1992) The neurobiology of selective attention. Current Biology 2: 165-169.

Posner, M.I., M.J. Nissen, and W.C. Ogden (1978) Attended and unattended processing modes: The role of set for spatial locations. In Modes of Processing and Perceiving Information, H.L. PICK, and I.J. SaltzMan, eds., pp. 137-157, Erlbaum, Hillsdale, NJ.

Posner, M.I., and S.E. Petersen (1990) The attention system of the human brain. Ann Rev Neurosci 13: 25-42.

Posner, M.I., C.R. Snyder, and B.J. Davidson (1980) Attention and the detection of signals. F Exp Psychol 109: $160-174$.

Post, L.J., and C.E. Chapman (1991) The effects of crossmodal manipulations of attention on the detection of vibrotactile stimuli in humans. Somatosens Mot Res 8: 149-157.

Ralston III, H.J., and D.D. Ralston (1994) Medial lemniscal and spinal projections to the macaque thalamus: An electron microscopic study of differing GABAergic circuitry serving thalamic somatosensory mechanisms. F Neurosci 14: 2485-2502.

Rees, G., C.D. Frith, and N. Lavie (1997) Modulating irrelevant motion perception by varying attentional load in an unrelated task. Science 278: 1616-1619.

Roland, P.E. (1982) Cortical regulation of selective attention in man. A regional cerebral blood flow study. $\mathcal{F}$ Neurophysiol 48: 1059-1078.

Sathian, K., and H. Burton (1991) The role of spatially selective attention in the tactile perception of texture. Percept Psychophys 50: 237-248.

Shiffrin, R.M., J.C. Craig, and E. Cohen (1973) On the degree of attention and capacity limitation in tactile processing. Percept Psychophys 13: 328-336.

Shiffrin, R.M., and D.W. Grantham (1974) Can attention be allocated to sensory modalities? Percept Psychophys 15: 460-474.

SHin, H.-C., and J.K. Chapin (1990a) Mapping the effects of SI cortex stimulation on somatosensory relay neurons in the rat thalamus: direct responses and afferent modulation. Somatosens Mot Res 7: 421-434.

Shin, H.-C., and J.K. Chapin (1990b) Movement induced modulation of afferent transmission to single neurons in the ventroposterior thalamus and somatosensory cortex in rat. Exp Brain Res 81: 515-522.

Shin, H.-C., H.J. Park, and J.K. Chapin (1993) Differential phasic modulation of short and long latency afferent sensory transmission to single neurons in the ventroposterolateral thalamus. Neurosci Res 17: $117-125$.
Steriade, M., L. Domich, and G. Oakson (1986) Reticularis thalami neurons revisited: activity changes during shifts of states in vigilance. 7 Neurosci 6: 68-81.

Sugitani, M. (1979) Electrophysiological and sensory properties of the thalamic reticular neurones related to somatic sensation in rats. F Physiol 290: 79-95.

Treisman, A.M. (1964) The effect of irrelevant material on the efficiency of selective listening. Am $\mathcal{F}$ Psychol 533-546.

Tremblay, N., M.C. Bushnell, and G.H. Duncan (1993) Thalamic VPM nucleus in the behaving monkey. II. Response to air-puff stimulation during discrimination and attention tasks. F Neurophysiol 69: 753-763.

Velasco, M., and F. Velasco (1975) Differential effect of task relevance on early and late components of cortical and subcortical somatic evoked potentials in man. Electroencephalogr Clin Neurophysiol 39: 353-364.

Velasco, M., F. Velasco, and A. Olvera (1980) Effect of task relevance and selective attention on components of cortical and subcortical evoked potentials in man. Electroencephalogr Clin Neurophysiol 48: 377-386.

WARREN, R.A., and E.G. Jones (1994) Glutamate activation of cat thalamic reticular nucleus: Effects on response properties of ventroposterior neurons. Exp Brain Res 100: 215-226.

Whang, K.C., H. Burton, and G.L. Shulman (1991) Selective attention in vibrotactile tasks: detecting the presence and absence of amplitude change. Percept Psychophys 50: 157-165.

WoldorfF, M.G., C.C. Gallen, S.A. Hampson, S.A. Hillyard, C. Pantev, D. Sobel, and F.E. Bloom (1993) Modulation of early sensory processing in human auditory cortex during auditory selective attention. Proc Natl Acad Sci USA 90: 8722-8726.

WoldorfF, M.G., and S.A. Hillyard (1991) Modulation of early auditory processing during selective listening to rapidly presented tones. Electroencephalogr Clin Neurophysiol 79: 170-191.

Yingling, C.D., and J.E. Skinner (1976) Selective regulation of thalamic sensory relay nuclei by nucleus reticularis thalami. Electroencephalogr Clin Neurophysiol 41: 476-482.

Yuan, B., T.J. Morrow, and K.L. Casey (1985) Responsiveness of ventrobasal thalamic neurons after suppression of $\mathrm{S} 1$ cortex in the anesthetized rat. 7 Neurosci 5 : 2971-2978.

Yuan, B., T.J. Morrow, and K.L. Casey (1986) Corticofugal influences of S1 cortex on ventrobasal thalamic neurons in the awake rat. F Neurosci 6: 3611-3617. 